\title{
Game Theory in an Age of Terrorism: How Can Statisticians Contribute?
}

\author{
Ronald D. Fricker, Jr. \\ Department of Operations Research, Naval Postgraduate School, \\ rdfricker@nps.edu
}

In The Law of Loopholes in Action [Gel05], David Gelernter argues that "every loophole will eventually be exploited; every loophole will eventually be closed." His thesis applied to terrorism means that terrorists will find security loopholes via continual exploration and that, once discovered, specific defensive measures have to be put in place to close each loophole.

The net effect of the Law of Loopholes, as anyone who flies regularly today knows, is an ever-expanding set of security rules and requirements. Such rules and requirements are useful for helping prevent the reoccurrence of a particular type of incident. But, when a determined adversary's focus is on causing general destruction and mayhem, then as one loophole is plugged, the adversary simply shifts its attention and energies to looking for and trying to exploit a different loophole.

The problem, of course, is that it is impossible to defend all potential targets (and their associated loopholes) against all threats all of the time. While it is important to implement certain new and improved defensive tactics, precisely because it is impossible to protect everything at all times, it is equally as important (and arguably more important) to implement offensive strategies to deter and disrupt these adversaries.

The question is, how to identify effective offensive and defensive strategies and tactics?

One approach is through the use of game theory, the mathematically based study and analysis of adversarial conflicts. The classic text The Compleat Strategyst [Wil66] characterizes games of strategy as having the following characteristics:

- A conflict: the participants (e.g., individuals, organizations, countries; known as "players" in game theory parlance) are at cross-purposes or have opposing interests.

- Adversarial reaction and interaction: each player has some control over the course of the conflict or its outcome via one or more decisions. 
- Outside forces: some aspects of the conflict are outside of the players' control and may be governed by chance or are unknown.

These characteristics clearly apply to the problem of thwarting terrorists and defeating terrorism.

The first extensive treatment of game theory was Theory of Games and Economic Behavior by John von Neumann and Oskar Morgenstern [VM44] in 1944. The seminal work on the subject, "Zur Theorie der Gesellschaftsspiele" by von Neumann [von28], was written in 1928. John von Neumann characterized the difference between games such as chess and games of strategy by saying "Chess is not a game. Chess is a well-defined form of computation. You may not be able to work out the answers, but in theory there must be a solution, a right procedure in any position. Now real games are not like that at all. Real life is not like that. Real life consists of bluffing, of little tactics of deception, of asking yourself what is the other man going to think I meant to do. And that is what games are about in my theory" [Pou92].

Game theoretic methods provide a structured way to examine how two adversaries will interact under various conflict scenarios. The results often provide insight into why real-world adversaries behave the way they do. In the middle and late 20th century, a great deal of game theoretic research focused on analyzing the arms race, nuclear brinkmanship, and Cold War strategies [Pou92]. While in the pre-9/11 era, game theory was also applied to terrorism, post-9/11 this work has expanded [SA03].

\section{Game Theory Applied to Terrorism}

In what is surely a gross oversimplification of the field (apologies to game theorists in advance), there are three broad categories of game theoretic methods applicable to the analysis of terrorism:

1. Classic games can generally be illustrated in a tabular form in which the players, their strategies, and their "payoffs" are completely specified. These types of games are often studied to determine whether there are a pair of strategies that result in an equilibrium between the two players (a "saddle point") and how the players will behave given the existence or absence of a saddle point.

2. Repetitive (or repeated) games, which are games that occur over time and the opponents repeatedly interact in a series of conflicts. These games are studied to gain insight into how players behave and react to their opponent's behavior and which behavioral strategies result in favorable or unfavorable final outcomes.

3. Tabletop games consisting of the simulation of an adversarial interaction with two or more actual (human) players using rules, data, and procedures designed to depict a conflict. "Tabletop" refers to the manner of older war games in which a battle was played out using miniature markers and 
maps on a table, much like the board game Risk. These types of games are generally less structured than the previous types, meaning the players have a much larger set of strategies available than can be easily tabularized.

Recent applications of game theoretic methods to the study of terrorism include: assessing strategies for how nations allocate expenditures for terrorism deterrence and the resulting implications for being attacked [AST87, SL68]; measures evaluating how various military employment policies/strategies encourage or discourage states from sponsoring terrorism [Art04]; assessing insurance risks via models that explicitly account for malicious terrorist intent [Maj02]; determining whether or not a stated policy of nonnegotiation with terrorist hostage-takers deters such behavior and under what conditions [LS88]; and evaluating the effects of focusing national antiterrorism policy on deterrence or prevention [SA03].

\section{Statistics and Game Theory}

In the parlance of game theory, much of classical statistics is a "one-person game" because there is no adversary. Classic statistical problems, particularly inferential problems, concern the estimation of an unobserved parameter or parameters. In these problems, the "adversary" is nature, manifested as randomness in some form or another, not as a willful opponent.

A frequent assumption in statistical methods, analyses, and models is that the parameter or population under study is fixed and the most important uncertainty to quantify is that which comes from sampling variability. Even in those problems where the parameter may change over time, the usual assumption is that the underlying mechanism that generates an outcome is unaffected by that outcome. (For example, in a regression model we assume the dependent variable does not or cannot affect the independent variable.) Neither of these assumptions is likely to be true in a game theory problem, where the population of interest is an intelligent adversary capable of changing its form, tactics, and responses.

The upshot is that most statisticians are not used to thinking about problems such as those addressed by game theory. However, statisticians are used to addressing problems in which uncertainty is either a natural component or must be quantified, and there is a lot of uncertainty in game theoretic models about deterring, detecting, and thwarting terrorists.

\section{How Can Statisticians Contribute?}

Game theoretic models tend to be fairly abstract models of reality. This has not prevented the models from providing useful insights into strategies for addressing certain types of conflicts, but it does lead to two specific questions: 
1. How well do the models fit observed data?

2. How can model uncertainty be quantified?

Both are questions that statisticians are well-suited to help address.

Possible ways statisticians could contribute to the further development of game theoretic methods, both in general and for terrorist problems in particular, include the following.

- Game theory models, including the strategies and their payoffs, are often defined in an ad hoc manner using expert judgment. A relevant statistical question is, how might data from past incidents and other knowledge be used to infer either the terrorist's "game" or the strategies they perceive or prefer? That is, how might a game be "fit" to observed data?

- The payoffs in game theory are utilities representing the desirability of the various outcomes to the players. In the absence of information, the utilities are often simply rankings of the various outcomes. A better methodology would be to elicit utilities from policymakers or subject-matter experts, much like one might elicit prior probabilities for a Bayesian analysis. Relevant questions include, what is (are) the best way(s) to elicit the utilities and how should utilities from multiple experts be combined?

- Once the payoffs are specified, the analysis of a game often treats them as fixed and known. How might the games be created, analyzed, and evaluated so that the uncertainty in payoffs is accounted for in the results, including the specification of the optimal strategy?

- Tabletop games are often useful for developing new insights and/or out-ofthe-box potential strategies, but they also often can only explore a small portion of the "game space." Relevant questions include how to characterize and account for the uncertainty in game design (e.g., a terrorist opponent's capabilities) and how statistical methods might be used to help design a series of games to best explore the "capabilities/strategy space."

- Finally, for new types of games that incorporate uncertainty, as well as for a set or series of more traditional games, how can graphical methods be employed to best display important game results, including appropriate depictions of uncertainty and variability?

The two chapters that follow this one discuss and examine how risk analysis can be combined with game theory. In "Combining Game Theory and Risk Analysis in Counterterrorism: A Smallpox Example," Banks and Anderson describe how to use risk analysis to generate random payoff matrices, which are then used to estimate the probability that a given strategy is optimal. In "Game-Theoretic and Reliability Methods in Counterterrorism and Security," Bier discusses the literature on reliability and risk analytic methods for rare events, game theory, and approaches for combining the two methods for defending complex systems against terrorist attack.

These two efforts represent a promising start towards addressing some of the problems described above. Yet more remains to be done. 


\section{References}

[Art04] Arthur, K. 2004. "Understanding the military's role in ending statesponsored terrorism." Master's thesis, Naval Postgraduate School.

[AST87] Atkinson, S. E., T. Sandler, and J. T. Tschirhart. 1987. "Terrorism in a bargaining framework." Journal of Law and Economics 30:1-21.

[Gel05] Gelernter, D. 2005. "The law of loopholes in action." Los Angeles Times, B13, May 6.

[LS88] Lapan, H. E., and T. Sandler. 1988. "To bargain or not to bargain: That is the question." American Economic Review 78:16-20.

[Maj02] Major, J. A. 2002. Advanced techniques for modeling terrorism risk. National Bureau of Economic Research Insurance Group Conference, February 1, 2002. http://www.guycarp.com/portal/extranet/pdf/major _terrorism.pdf, downloaded on June 30, 2005.

[Pou92] Poundstone, W. 1992. Prisoner's dilemma. New York: Doubleday.

[SA03] Sandler, T., and D. G. Arce M. 2003. "Terrorism and game theory." Simulation and Gaming 34:319-337.

[SL68] Sandler, T., and H. E. Lapan. 1968. "The calculus of dissent: An analysis of terrorists' choice of targets." Synthese 76:245-261.

[von28] von Neumann, J. 1928. "Zur Theorie der Gesellschaftsspiele." Mathematische Annalen 100:295-300.

[VM44] von Neumann, J., and O. Morgenstern. 1944. Theory of games and economic behavior. Princeton, NJ: Princeton University Press.

[Wil66] Williams, J. D. 1966. The compleat strategyst, rev. ed. New York: McGraw-Hill Book Company. 